\title{
Paramagnetic to antiferromagnetic phase transformation in sputter deposited Pt-Mn thin films
}

\author{
Peter F. Ladwiga) and Y. Austin Chang \\ Materials Science Program, University of Wisconsin-Madison, Madison, Wisconsin 53706 \\ Eric S. Linville, Augusto Morrone, Jenny Gao, Bharat B. Pant, Alan E. Schlutz, \\ and Sining Mao \\ Recording Head Operations, Seagate Technology, Bloomington, Minnesota 55435
}

(Received 12 December 2002; accepted 8 May 2003)

\begin{abstract}
Sputter deposited, equiatomic Pt-Mn thin films used in giant magnetoresistive spin valves are found not to exist in the antiferromagnetic state required for device operation. Therefore, an annealing step is needed to induce a phase transformation from the as-deposited, paramagnetic $A 1$ ( $\mathrm{fcc}$ ) phase to the antiferromagnetic $L 1_{0}$ phase. The $L 1_{0}$ phase is the thermodynamically stable configuration, but favorable kinetics for the transformation were only found above $260^{\circ} \mathrm{C}$. The $A 1$ to $L 1_{0}$ phase transformation was studied by $\mathrm{x}$-ray diffraction, transmission electron microscopy and differential scanning calorimetry (DSC). The nucleation and growth conditions were evaluated and an exothermic transformation enthalpy of $-12.1 \mathrm{~kJ} / \mathrm{mol}$ of atoms was determined. The kinetics of the reaction were simulated using the Johnson-Mehl-Avrami analysis, where the necessary parameters were determined by the Kissinger and Ozawa methods from constant scanning rate DSC experiments [H. Yinnon and D. R. Uhlmann, J. Non-Cryst. Solids 54, 253 (1983)]. The resulting simulations were compared to DSC data as well as isothermal x-ray peak shift data and a reasonable agreement was obtained. (C) 2003 American Institute of Physics. [DOI: 10.1063/1.1587266]
\end{abstract}

\section{INTRODUCTION}

The dramatic increase in the areal density of magnetic disk drives has been driven by the discovery of giant magnetoresistance (GMR) and its incorporation into spin valve read heads. ${ }^{1-7}$ These sensors make use of the GMR effect by varying their electrical resistance depending on the local magnetic state of the information storage media. Due to their large change in resistance, spin valve sensors are needed to meet the current areal density requirements sought after by the mass storage industry.

A significant material challenge in engineering spin valve read heads is finding an antiferromagnetic (AFM) pinning material that simultaneously meets all of the requirements to ensure stable operation of the device and is relatively simple to process. $^{8}$ Some of these requirements include a high pinning field at the operating temperature of the device (approximately $120^{\circ} \mathrm{C}$ ), ${ }^{9}$ a high blocking temperature (i.e., the temperature at which the pinning field drops to zero), good corrosion resistance, and low cost. One class of AFM materials that can meet these requirements are the $L 1_{0}$ ordered tetragonal alloys, ${ }^{8,10-14}$ such as NiMn and PdMn. Another material from this class, PtMn, has emerged as a promising candidate for exchange biasing., ${ }^{8,15-19}$

There is a significant processing obstacle that arises in using PtMn as the AFM; the sputter deposited form of PtMn is not antiferromagnetic but is rather a paramagnetic, chemically disordered, face-centered-cubic $(A 1)$ phase. A postdeposition anneal is needed to transform PtMn to the desired antiferromagnetic, $L 1_{0}$ phase. The objective of this study is

\footnotetext{
${ }^{\text {a)} E l e c t r o n i c ~ m a i l: ~ p f l a d w i g @ s t u d e n t s . w i s c . e d u ~}$
}

to investigate the thermodynamics and kinetics of the PtMn phase transformation from the metastable $A 1$ phase obtained by sputter deposition to the stable, antiferromagnetic $L 1_{0}$ phase using x-ray diffraction (XRD), transmission electron microscopy (TEM), and differential scanning calorimetry (DSC).

The phase transformation anneal can have detrimental effects on other parts of the sensor, such as interdiffusion and magnetic realignment, which are minimized by lower temperatures and shorter anneal times. By incorporating the Johnson-Mehl-Avrami analysis, a model was created which can be used to find the ideal annealing conditions to complete the phase transformation while minimizing damage to other parts of the sensor.

\section{EXPERIMENT}

The samples used in the experiments consist of PtMn and ferromagnetic layers dc magnetron sputter deposited from compound targets onto thermally oxidized $\mathrm{Si}$ substrates, with a thin seed layer preceding the stack deposition. The purpose of the seed material is to induce a desired (111) texture in the subsequent layers. The ferromagnetic layers used in the experiments were permalloy $\left(\mathrm{Ni}_{81} \mathrm{Fe}_{19}\right.$ wt. \%, henceforth referred to as $\mathrm{Ni}-\mathrm{Fe}$ ) and a single phase, $A 1$ solid solution of a few percent of $\mathrm{Fe}$ in $\mathrm{Co}$ (henceforth termed $\mathrm{Co}-\mathrm{Fe}$ ).

The XRD and TEM samples consist of single antiferromagnet/ferromagnet bilayers with a PtMn layer thickness of $250 \AA$. These samples were also capped with 50 $\AA$ of the seed material to protect the bilayer during annealing. The films were annealed in a furnace at $260^{\circ} \mathrm{C}$ in order 


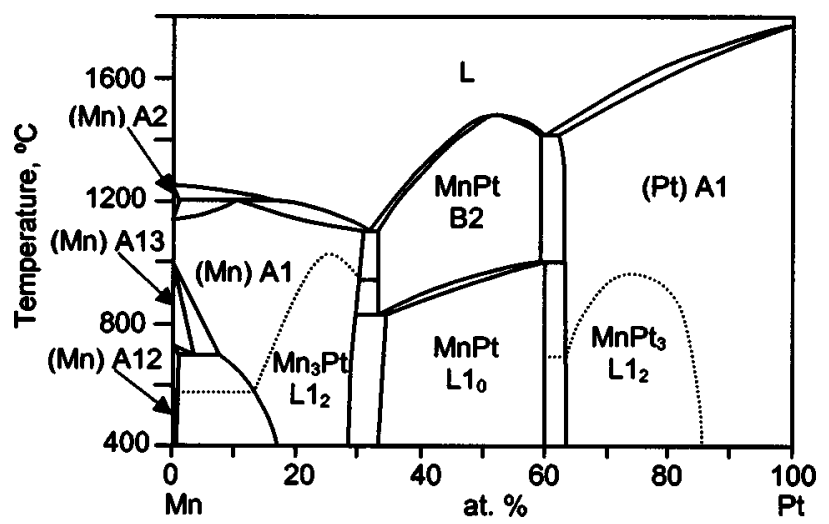

FIG. 1. The Pt-Mn bulk equilibrium phase diagram by Mahler (See Ref. 21). The three phases present in the equiatomic region are liquid, $B 2$ and $L 1_{0}$ phases.

to induce various states of order in the PtMn layer. The ramp and cool profiles of the furnace were set to $3{ }^{\circ} \mathrm{C} / \mathrm{min}$ and the anneal times given are without the addition of ramp and cool times. The background pressure of the furnace was 1 mTorr, and during annealing the chamber was backfilled with 1 Torr of $\mathrm{N}_{2}$. The structural characterization was done with a Phillips Xpert x-ray diffractometer utilizing $\mathrm{Cu} K_{\alpha}$ radiation and a JEOL $2010200 \mathrm{keV}$ TEM.

The samples used for DSC experiments consisted of 74 repeats of the antiferromagnet/ferromagnet bilayer with 150 $\AA$-thick PtMn layers. In order to produce good signal to noise ratios in the DSC, $10-20 \mathrm{mg}$ of the repeating bilayer stack were needed. This necessitated that the film be removed from the Si substrate. ${ }^{20}$ This was accomplished by coating the $\mathrm{Si}$ wafers with a polymer release layer prior to deposition. Most of the film was mechanically peeled away from the polymer, while the remaining film was removed by dissolving the polymer layer away in acetone. To fully remove the traces of polymer residue, over 20 soak/filter/rinse cycles were performed with acetone and then ethanol for the final few cycles. The cleansed film was air dried at room temperature for 2 days before initiating the DSC experiments. The DSC experiments were carried out using a Perkin Elmer DSC 7 with Pyrus series software. The atmosphere consisted of $99.998 \%$ pure Ar passed through a Centorr Associates $\mathrm{Ti}$ deoxidizing furnace before being sent to the sample and reference chambers at $25 \mathrm{~cm}^{3} / \mathrm{min}$ (verified by individual Ar calibrated flowmeters). The system was purged at $50^{\circ} \mathrm{C}$ for at least $1 \mathrm{~h}$ after sample insertion. Both $\mathrm{Al}$ and $\mathrm{Cu}$ pans were used and no sample-pan reactions were observed below $500{ }^{\circ} \mathrm{C}$. Since no reversible peaks were observed, each sample was reheated and the second trace subtracted from the first to assure a flat base line.

\section{RESULTS AND DISCUSSION}

\section{A. Phase equilibria investigation}

The Pt-Mn phase diagram by Mahler ${ }^{21}$ (Fig. 1) shows three polymorphs at the equiatomic concentration: a high temperature liquid phase; a mid-temperature, chemically ordered, $B 2$ phase; and a low temperature, chemically ordered, antiferromagnetic, $L 1_{0}$ phase. The $A 1$ (fcc) structure ob- tained by sputter deposition is metastable and does not appear on the equilibrium phase diagram at the equiatomic concentration. However, it is possible to see how the metastable $A 1$ phase could form. If the $B 2, L 1_{0}$, and $L 1_{2}$ ordered phases were suspended from the phase diagram, the adjacent $\mathrm{Pt}$ and $\mathrm{Mn}$ phases are both A1 and would likely join as visualized by drawing metastable extensions. ${ }^{22}$ During sputter deposition, vapor atoms condense to form solid thin films. The thermodynamic effect is a rapid removal of the heat of sublimation. The kinetic effect is a quench rate that is too fast to allow the formation of the ordered PtMn structures and the chemically disordered $A 1$ phase forms. ${ }^{23}$ This metastable phase will transform to the stable $L 1_{0}$ phase with increasing time and temperature. Therefore, the anneal required to transform the metastable $A 1$ phase to the stable $L 1_{0}$ phase is necessary only for kinetic reasons. There is no thermodynamic boundary to overcome by heating; the anneal just provides the necessary activation energy for atomic rearrangement. Furthermore, the irreversible nature of the heat signals from DSC scans shown later gives sufficient evidence that the $L 1_{0}$ phase is indeed the stable phase in the thin film samples investigated here.

\section{B. TEM and electron diffraction}

The observed microstructure consists of three primary types. The first is the as-deposited $A 1$ structure shown in Fig. 2 , which consists of many nanotwin blocks randomly distributed throughout the layer. The $\mathrm{Ni}-\mathrm{Fe}$ layer also contains nanotwin defects, but to a much smaller degree. Figure 2 also shows a selected area diffraction pattern within the region of the image, which indicates the sample is oriented along a (110) zone axis. Two sets of diffraction spots are observed, which are from the seed layer (outside set) and the PtMn layer (inside set). Twin spots are observed for both the PtMn and seed layer. Streaking is also observed perpendicular to the sample normal, which indicates that the twin defects are closely spaced and stacking along the film growth direction. These nanotwin defects may act as preferential nucleation sites for the $L 1_{0}$ phase.

The second type of microstructure consists of recently nucleated $L 1_{0}$ phase growing into the $A 1$ matrix, as shown in Fig. 3 after an anneal at $260^{\circ} \mathrm{C}$ for $2 \mathrm{~h}$ and $55 \mathrm{~min}$. In the upper image, the as-deposited $A 1$ microstructure is clearly present on the left side of the sample. Arrows indicate two nucleated $L 1_{0}$ PtMn particles separated by a region of $A 1$ phase. Twinning is clearly observed in the left $L 1_{0}$ particle. The bottom, high-resolution image shows the twinning in more detail. The twin plane is a (111) plane and the lattice fringes in each of the twinned regions are clearly visible. The angle between the twin plane and the lattice fringes is approximately $68^{\circ}$, which suggests that all the visible fringes in this region are from (111) planes. The reorientation of this particle is approximately $32^{\circ}$, as calculated from the angle between the sample normal and the (111) twin plane. The $A 1$ structure on the left shows a much more continuous nanotwin structure as opposed to the block-type nanotwin structure observed in the majority of the regions. This may contribute to the ability of PtMn to reorient in this region. 

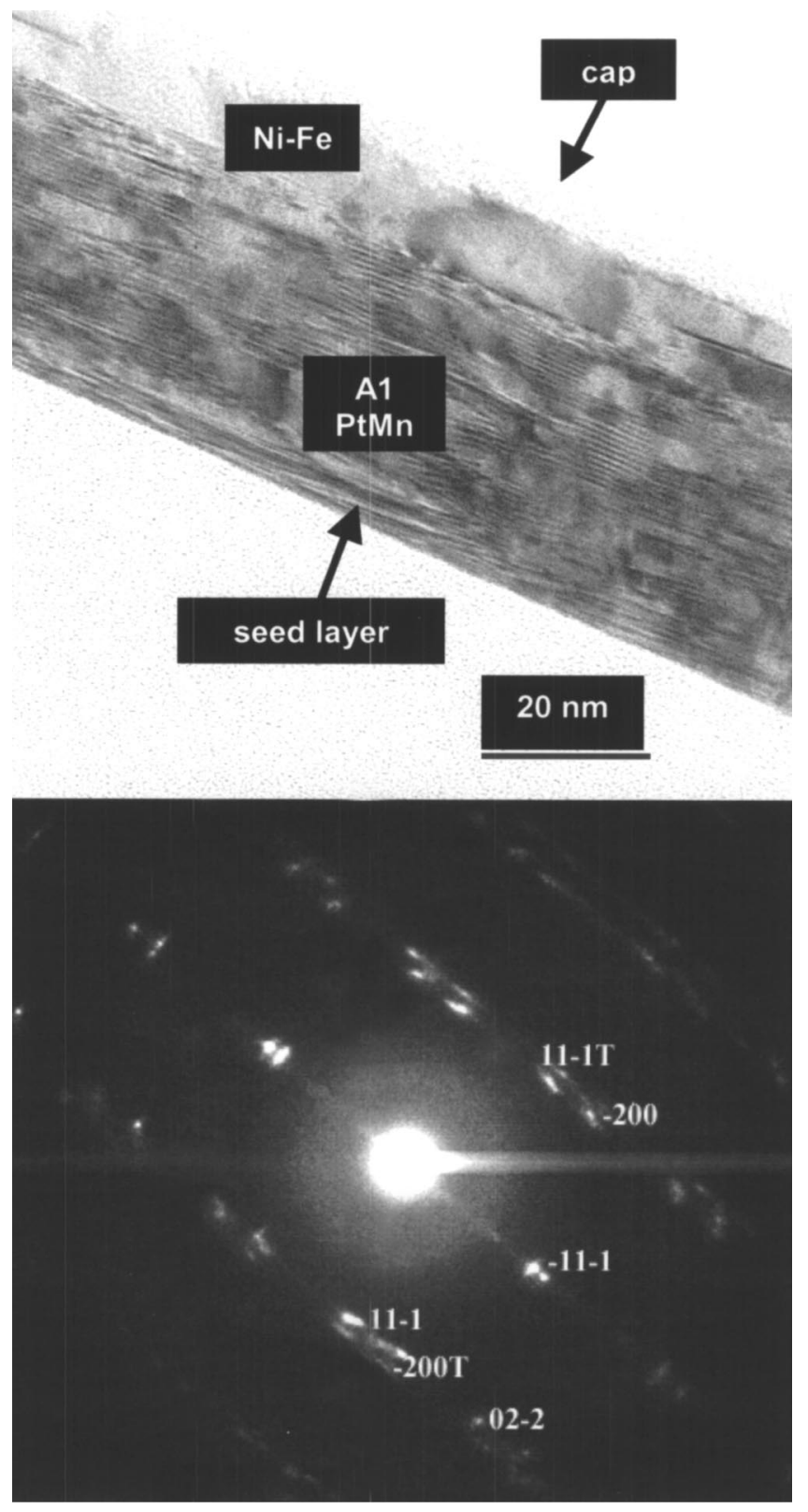

FIG. 2. Bright-field image and selected area diffraction pattern of asdeposited PtMn/Ni-Fe.

Furthermore, the presence of a grain boundary in the $\mathrm{Ni}-\mathrm{Fe}$ layer above the $L 1_{0}$ particle suggests that nucleation took place at a grain boundary in the preexisting $A 1$ structure.

The final type of microstructure observed in these samples consists of significantly transformed regions of $L 1_{0}$ PtMn oriented in a (111) texture, as shown in Fig. 4. In this case, the $L 1_{0}$ PtMn phase nucleates and quickly grows laterally (relative to the perpendicular direction) as a thin plate until it is impeded by grain boundaries or defects. It then grows perpendicular to the film growth direction from both sides. In Fig. 4 , the $L 1_{0}$ particle likely nucleated somewhere above the center of the film and it is growing into the $A 1$ phase below. This high-resolution image shows that the $L 1_{0}$ PtMn is (111) textured to match the parent phase, but it lacks the block nanotwin defects present in the $A 1$ phase.
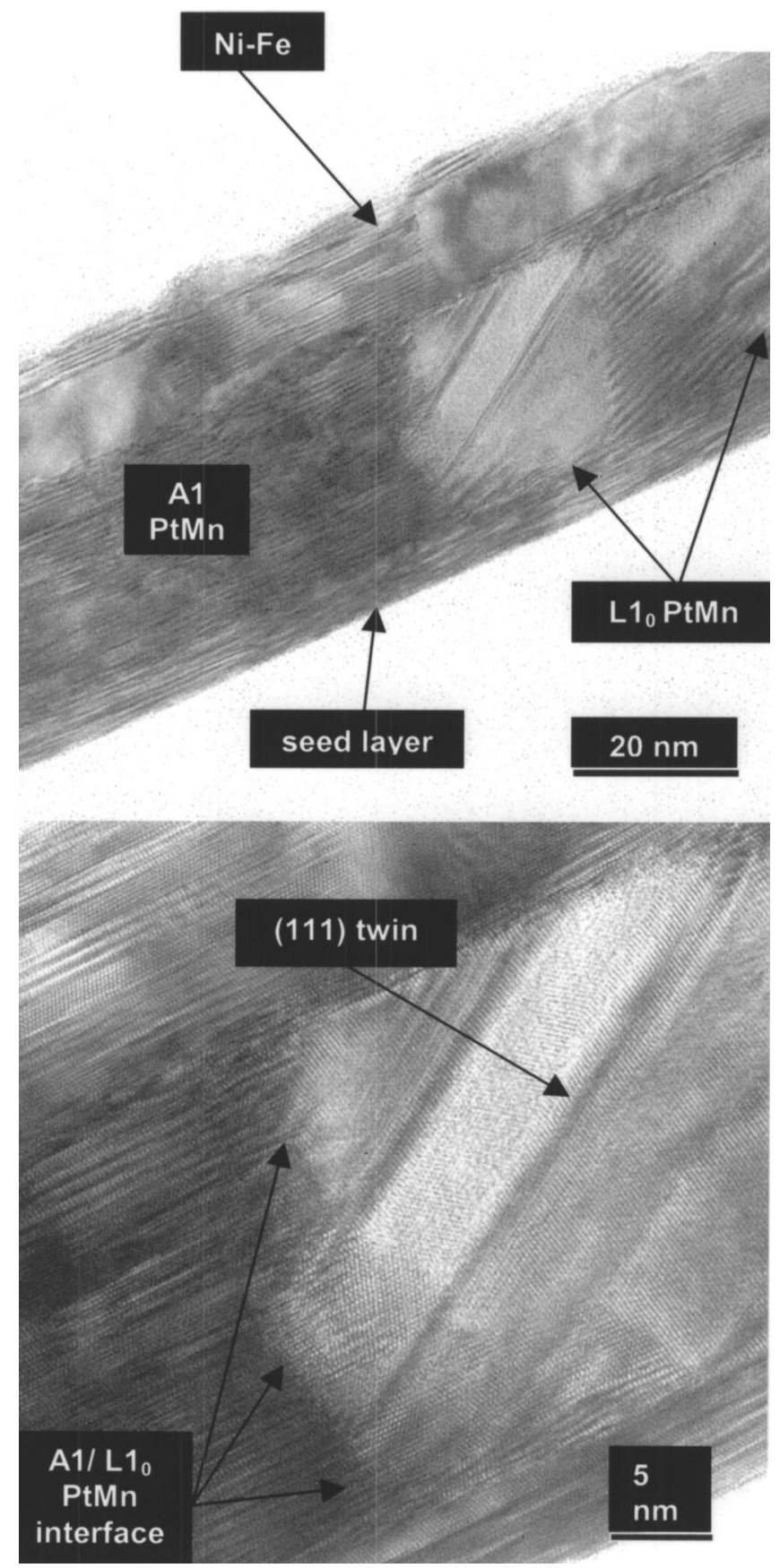

FIG. 3. Bright-field TEM image of the PtMn/Ni-Fe sample annealed at $260^{\circ} \mathrm{C}$ for $2 \mathrm{~h}$ and $55 \mathrm{~min}$.

As we move from samples that are in an intermediate stage of phase transformation to those that are closer to completion, the $A 1$ phase is largely absent from the samples, along with its characteristic block nanotwin microstructure. Figure 5 shows a bright-field TEM image from the sample annealed at $260^{\circ} \mathrm{C}$ for $6 \mathrm{~h}$. This region consists of two welldefined $L 1_{0}$ particles that have grown to both the $\mathrm{Ni}-\mathrm{Fe}$ and seed layer interfaces. The left particle is (111) textured, and (111) twins running from interface to interface are clearly visible. These twins are a variant of the (111) twins that are seen parallel to the film plane. The arrow points to a twin in the left particle that has grown to the interface between the two $L 1_{0}$ particles. It is clearly seen that this twin is unable to 


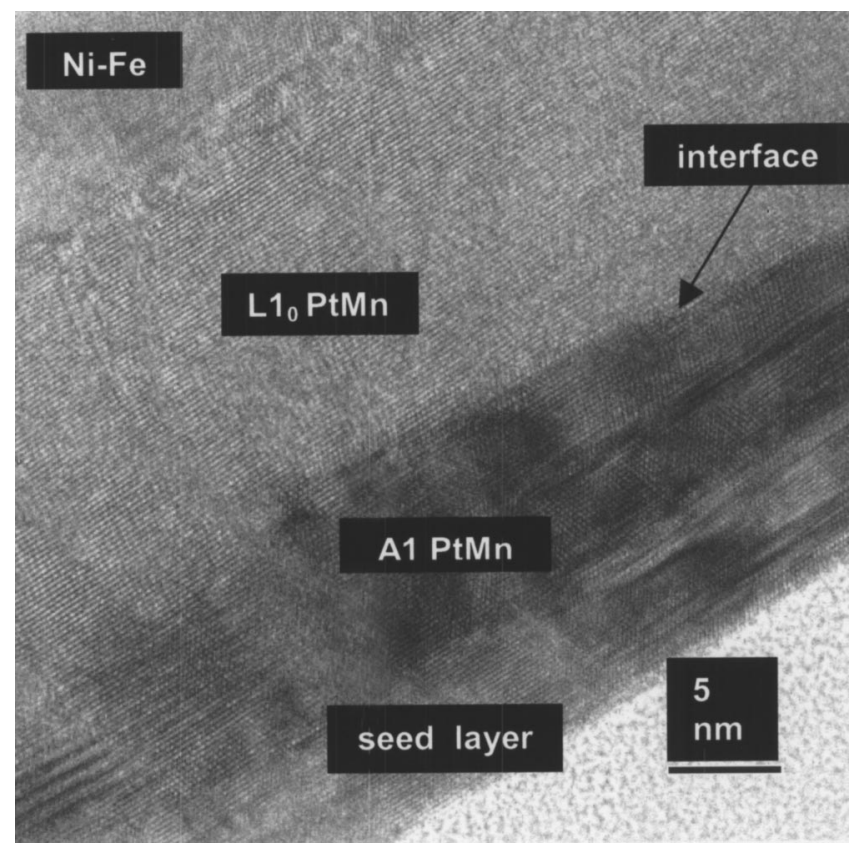

FIG. 4. Bright-field TEM image of the PtMn/Ni-Fe sample annealed at $260{ }^{\circ} \mathrm{C}$ for $2 \mathrm{~h}$ and $55 \mathrm{~min}$.

penetrate this interface and its growth is impeded at this point.

The microstructure of the sample annealed at $260{ }^{\circ} \mathrm{C}$ for $8 \mathrm{~h}$ was very similar to that of the sample annealed for $14 \mathrm{~h}$ where the samples were nearly fully transformed. In fully transformed, (110) zone, selected area diffraction patterns (not shown) the angle between (111) diffraction spots was measured as $68.1^{\circ}$, consistent with the $L 1_{0}$ phase of PtMn.

In short, the $A 1-L 1_{0}$ transformation occurs by the nucleation of ordered clusters in earlier stages of the transformation based on TEM images that do not show any evi-

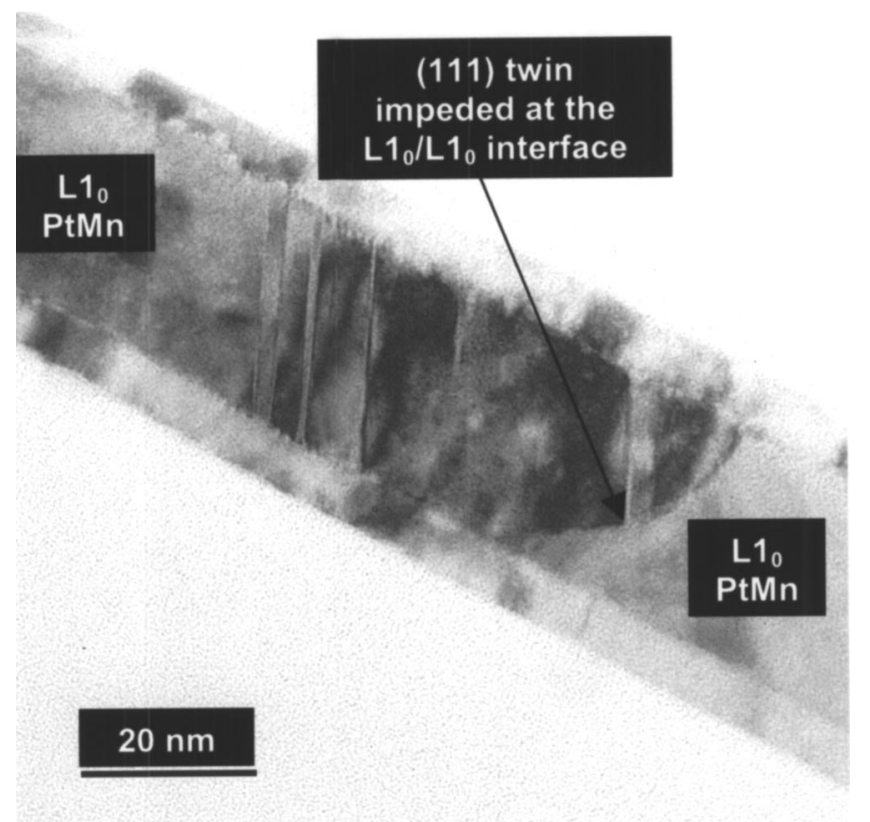

FIG. 5. A bright-field TEM image from a PtMn/Ni-Fe sample annealed at $260^{\circ} \mathrm{C}$ for $6 \mathrm{~h}$

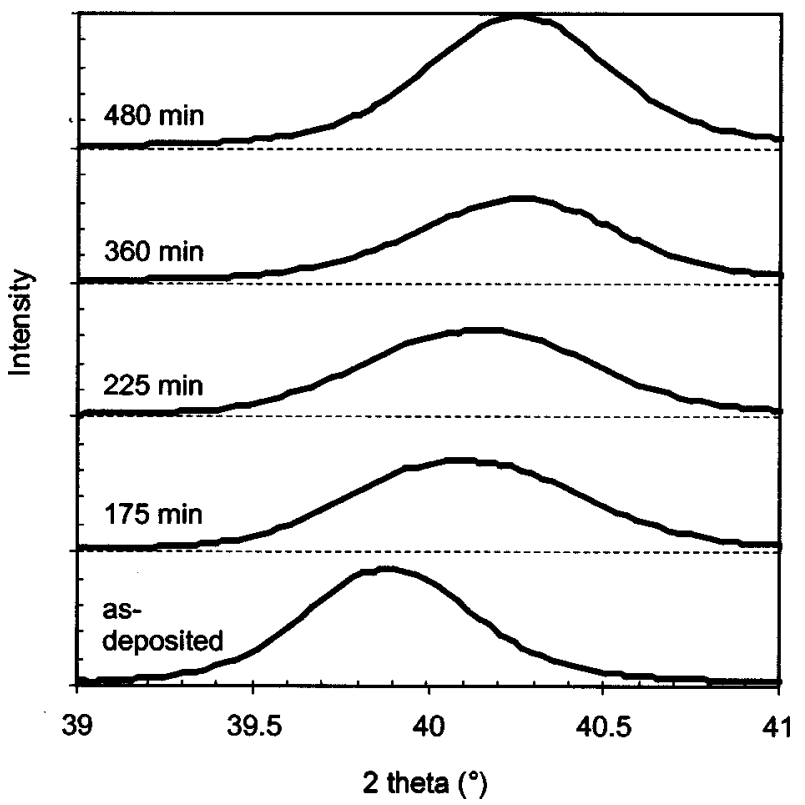

FIG. 6. X-ray Bragg scan results illustrating the (111) peak shift as the sample transforms from the as-deposited $A 1$ phase to the antiferromagnetic $L 1_{0}$ phase at $260^{\circ} \mathrm{C}$. Annealing times are given in minutes.

dence of newly nucleated clusters after the PtMn layer is about $40 \%$ transformed. TEM images suggest that grain boundaries and areas with a high concentration of nanotwin defects may serve as preferential nucleation sites. The typical nucleated cluster grows along (111) twins until impingement and then grows laterally. Some postimpingement coarsening is also observed at long annealing times.

\section{X-ray diffraction}

Figure 6 shows the sequence of Bragg x-ray diffraction scans for an as-deposited sample as well as for annealing times of $175,225,360$, and $480 \mathrm{~min}$ at $260^{\circ} \mathrm{C}$. The slight peak shift corresponds to the superposition of separate $A 1$ and $L 1_{0}$ (111) planar spacing peaks. The overall shift is minimal; only a two-theta shift from 39.88 to 40.25 is observed as the ratio of $A 1-L 1_{0}$ phase decreases. The full width at half maximum peak breadth reaches a maximum at approximately $50 \%$ transformed phase, and then decreases to a value similar to the as-deposited state as the phase transformation completes. This is due to the superposition of the $A 1$ and $L 1_{0}$ phases that contribute to the observed (111) PtMn peak.

While the phase transformation is taking place, the decrease in diffracted peak intensity is due to the superposition of separated peaks but is also due to a small degree of reorientation from the (111) texture described earlier. TEM images suggest that the (111) textured $L 1_{0}$ phase that dominates the growth consumes the small amount of reoriented $L 1_{0}$ phase present after completion of the phase transformation. As the reoriented $L 1_{0}$ texture is consumed by the (111) textured $L 1_{0}$ phase late in the transformation, the $L 1_{0}$ peak intensity increases accordingly. Thus, the final $L 1_{0}$ phase consists primarily of (111) texture that matches the parent phase. 


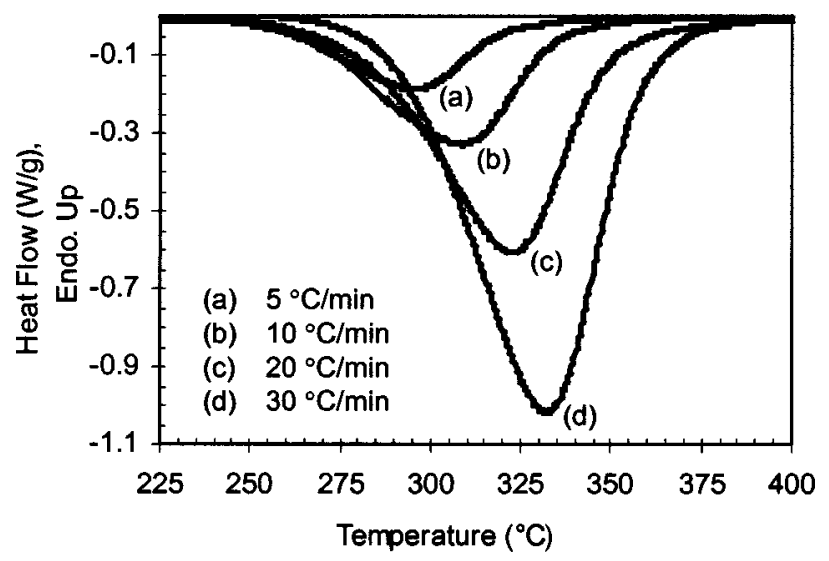

FIG. 7. DSC results from four separate scanning rates. The integrated areas under each peak (measured in $\mathrm{J} / \mathrm{g}$ ) are quite similar with a standard deviation under $5 \%$.

The shift in two-theta with annealing time was used to give an approximate measure of the degree of phase transformation in these samples (data presented later in Fig. 10). The termination of the two-theta shift marks the completion of the phase transformation. Approximately $45 \%$ of the PtMn film is transformed to $L 1_{0}$ after annealing for $175 \mathrm{~min}$ at $260{ }^{\circ} \mathrm{C}$. After $6 \mathrm{~h}$ the phase transformation is nearly complete, but it takes over $8 \mathrm{~h}$ to fully saturate the two-theta value. This shows that the samples are difficult to fully transform at $260^{\circ} \mathrm{C}$, and long annealing times are required. It is important to note that the XRD peak shift correlation to degree of phase transformation is merely an approximation, due to the complications of reorientation and particle size that certainly have effects on the intensity and breadth of the individual contributions to the observed peak.

\section{Differential scanning calorimetry}

The differential scanning calorimeter (DSC) measures heat flow into/out of a sample. The energy is quantified by comparison to an unreactive reference, and the system can be operated in isochronal (constant heating rate) or isothermal mode. ${ }^{20}$ The time-temperature relationship in an isochronal experiment is given by Eq. (1):

$$
T=T_{0}+Q t,
$$

where $T$ is temperature, $T_{0}$ is the initial temperature of the experiment, $Q$ is the heating rate, and $t$ is time. DSC results from four isochronal experiments are shown in Fig. 7, which shows exothermic heat signals obtained as the $\mathrm{PtMn} / \mathrm{Co}-\mathrm{Fe}$ multilayer is heated at $5,10,20$, and $30^{\circ} \mathrm{C} / \mathrm{min}$. From these and other DSC scans, we will extract thermodynamic and kinetic parameters needed to model the phase transformation.

The onset temperature of the reaction is calculated by drawing a tangent to the peak at the point of minimum (largest negative value) slope and finding the point at which it intersects the baseline. Due to the shift to higher temperatures with heating rate, the onset temperature was calculated from a $5{ }^{\circ} \mathrm{C} / \mathrm{min}$ scan, the rate at which the DSC used in this study was calibrated with In and $\mathrm{Zn}$ standards. The onset temperature calculated from the $5{ }^{\circ} \mathrm{C} / \mathrm{min}$ scan in Fig. 7(a) is $260.6^{\circ} \mathrm{C}$. This explains the slow transformation kinetics depicted in the previously described $260^{\circ} \mathrm{C}$ anneal.

The onset temperature for the $A 1-L 1_{0}$ phase transformation does not have the thermodynamic meaning traditionally associated with stable phase transformations. Because the reaction represents a transformation from a metastable to the stable structure, the onset temperature does not represent a precise limit defining a phase boundary between $A 1$ and $L 1_{0}$ structures, but rather serves as a relative indicator between regions of fast and slow reaction kinetics. In this respect, this phase transformation in PtMn is quite similar to the crystallization of metallic glasses, which is extensively studied with DSC.

The enthalpy of transformation was calculated from the DSC scans as well. The total area of the exothermic peak gives the magnitude of the heat given off during the reaction. Since temperature in an isochronal scan is directly related to time and the heat flow traces are normalized by sample weight, all four scans can be used to calculate the enthalpy of the transformation, although higher rate scans typically have better signal to noise. The average heat flow from the multilayers is $-88.9 \mathrm{~J} / \mathrm{g}$ with a standard deviation of $4.4 \%$. However, assuming that all of the heat flow is a result of transformation within the PtMn layers, which encompass 92 wt \% of the multilayer sample, the enthalpy of the $A 1$ to $L 1_{0}$ phase transformation is $-96.6 \mathrm{~J} / \mathrm{g}$, or $-12.1 \mathrm{~kJ} / \mathrm{mol}$ of atoms. At first inspection, this value may appear somewhat higher than expected, being that many compounds have enthalpies of formation on this order of magnitude. Unfortunately, the necessary thermodynamic data for PtMn does not exist and this value cannot be crosschecked with thermodynamic calculations. However, the enthalpy of formation of PtMn calculated from semi-empirical models ranges from -42 to $-43.9 \mathrm{~kJ} / \mathrm{mol}$ of atoms. ${ }^{24,25}$ It is expected that the measured order-disorder phase transformation enthalpy in this study is lower than the calculated enthalpy of formation of the compound from pure elements.

The fraction transformed at any point along the DSC scan can easily be calculated with the assumption that the magnitude of the DSC signal is directly proportional to the amount of material transforming from $A 1$ to $L 1_{0}$. Integration over a given time interval divided by the total enthalpy yields the fraction transformed, $x$, during that interval. The fraction transformed over a given time interval can also be calculated from isochronal scans, taking into account the time-temperature correlation given in Eq. (1). These datasets will be shown later in conjunction with phase transformation modeling results (Figs. 9 and 10).

\section{E. Phase transformation modeling}

With the contention that the $A 1-L 1_{0}$ phase transformation in PtMn is a suitable nucleation and growth reaction, we can now employ the Johnson-Mehl-Avrami (JMA) analysis to model the phase transformation. ${ }^{20,26-36}$ The JMA analysis makes assumptions that enable the simulation of the phase transformation over a range of time-temperature combinations. Henderson ${ }^{26}$ has published a critical analysis on the applicability of the JMA analysis to nonisothermal condi- 
tions. The primary assumption of the JMA theory is that the transformation rate depends only on the state variables of fraction transformed and time (i.e., independent of thermal history). ${ }^{26,27}$ Henderson describes the ideal candidate for nonisothermal application of JMA to be a reaction that only undergoes a morphological structure change and a reaction in which the nucleation process takes place very early in the transformation, i.e., point site saturation. ${ }^{26}$ The polymorphic nature of the PtMn phase transformation has been established, and TEM images support the claim that the nucleation takes place early in the transformation although they cannot confirm point site saturation. Ultimately, the validity of these assumptions to this particular transformation will be tested by comparison to the XRD and DSC results.

The JMA analysis contends that a nucleation and growth transformation can be fit to

$$
x=1-\exp \left[-(K \cdot t)^{n}\right],
$$

where $x$ is the fraction transformed, $K$ is a rate constant with dimensions of time ${ }^{-1}, t$ is time, and $n$ is a dimensionless parameter known as the Avrami exponent. The rate constant is assumed to have an Arrhenian temperature dependence

$$
K=K_{0} \exp \left(-E_{a} / R T\right),
$$

which is strictly incorrect but serves as a useful approximation over the limited range of temperatures in which the phase transformation will be analyzed. ${ }^{26-28,37}$

With the addition of the Kissinger analysis, the activation energy associated with the transformation can be calculated. Although the analysis was originally derived to describe a limited set of reactions, ${ }^{38}$ its applicability has expanded and it is now used for both nucleation and growth, ${ }^{20,26,27,29,39-41}$ and grain growth reactions. ${ }^{28}$ Different approaches can be used to derive the relationship which claims that for thermally activated reactions there exists a linear dependence between $\ln \left(Q / T_{p}^{2}\right)$ and $1 / T_{p}$, where $T_{p}$ is the peak temperature. The proportionality constant or slope is the activation energy, $E_{a}$, normalized by the gas constant, $R$ :

$$
\frac{d \ln \left(Q / T_{P}^{2}\right)}{d 1 / T_{P}}=-\frac{E_{a}}{R} .
$$

When the Kissinger analysis is applied to the PtMn DSC results, the resulting $E_{a}$ is $131.5 \mathrm{~kJ} / \mathrm{mol}$, calculated with a linear correlation coefficient $\left(r^{2}\right)$ of 0.999 .

It must be noted that this calculated activation energy is the overall activation energy for the entire phase transformation process ${ }^{27,42}$ including nucleation and growth of the $L 1_{0}$ phase. Furthermore, it is worthwhile to perform some rough calculations to see whether this activation energy lies within the acceptable application boundaries of the classical nucleation theory. The classical nucleation theory generally applies to reactions whose activation energy lies between approximately 15 and 60 times the average thermal energy, ${ }^{43}$ given by $R \cdot T$. The upper limit of approximately $60 R T$ is a practical limit in which the concentration of critical sized clusters is too small for appreciable nucleation. The lower limit of approximately $15 R T$ defines the region where nucleation rates are so high that the classical nucleation theory is

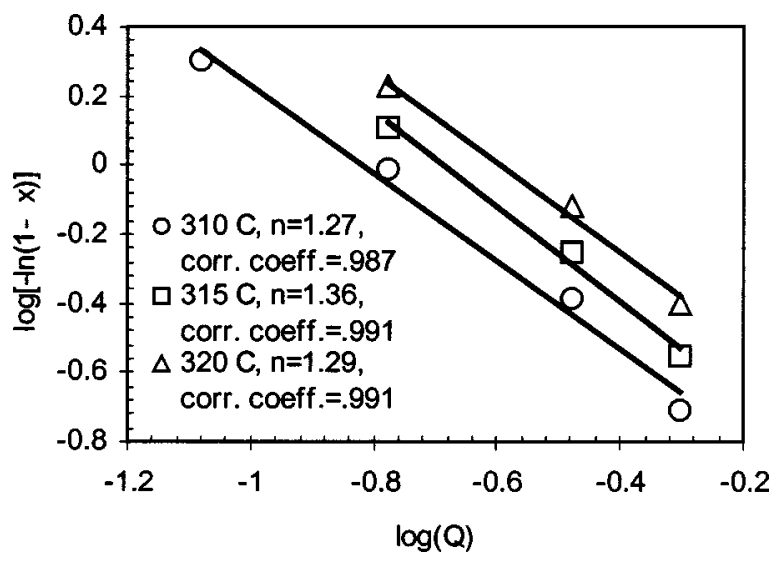

FIG. 8. Ozawa analysis plots. The data was taken from DSC scans at 310, 315 , and $320^{\circ} \mathrm{C}$. The negative slope of each line is used to determine the Avrami exponent.

no longer applicable. Comparing $E_{a}$ to the average thermal energy at the onset temperature of $260.6^{\circ} \mathrm{C}$, we find that $E_{a}=30 R T$; therefore, the contention that the DSC peak represents a nucleation and growth reaction seems valid.

The Avrami exponent, $n$, can be calculated from isochronal experiments with a mathematical manipulation of Eqs. (1) and (2), first suggested by Ozawa, ${ }^{44}$ but more recently described by Yinnon and Uhlmann. ${ }^{27}$ Substituting Eq. (1) into Eq. (2) to replace $t$, the Avrami exponent is obtained by differentiating

$$
\log [-\ln (1-x)]=n \log \left[K\left(T-T_{0}\right)\right]-n \log Q
$$

at constant $T$ to yield

$$
\left.\frac{d\{\log [-\ln (1-x)]\}}{d \log Q}\right|_{T}=-n .
$$

Figure 8 shows the results of Ozawa analyses at three separate temperatures, with the average $n$ calculated as 1.31. A benefit of this analysis in determining $n$ is that it makes no further assumptions other than those involved in Eq. (2). ${ }^{27}$ The value of $n$ reflects a convolution of contributing factors such as nucleation sites, growth mode and the dimensionality of growth. ${ }^{28,34,39,45}$ Low values of $n$, as is the case here, represent a significant departure from spatially random, constant nucleation rate conditions. ${ }^{28,34,35,46,47}$ The TEM images suggest that preferred nucleation sites exist and that the nucleation of $L 1_{0}$ particles occurs predominantly during the early stages of the transformation. These factors support the calculation of a low Avrami exponent.

Using the values determined for $E_{a}$ and $n$, the last independent parameter, $K_{0}$, is calculated from the best fit to the isochronal DSC data. From the DSC scans shown in Fig. 7, $\ln \left(K_{0}\right)=19.95$. Plots of $x$ vs $T$ from experimental data and simulations are shown in Fig. 9. On the whole, the simulations agree very well with the experimental data. However, there is a consistent deviation from the experimental data at high fraction transformed. There are several possible explanations which all may contribute in varying degrees. First is the nature of the DSC apparatus. There is an unavoidable thermal lag produced due to the time it takes for heat taken into/given off from the sample to reach the furnace thermo- 


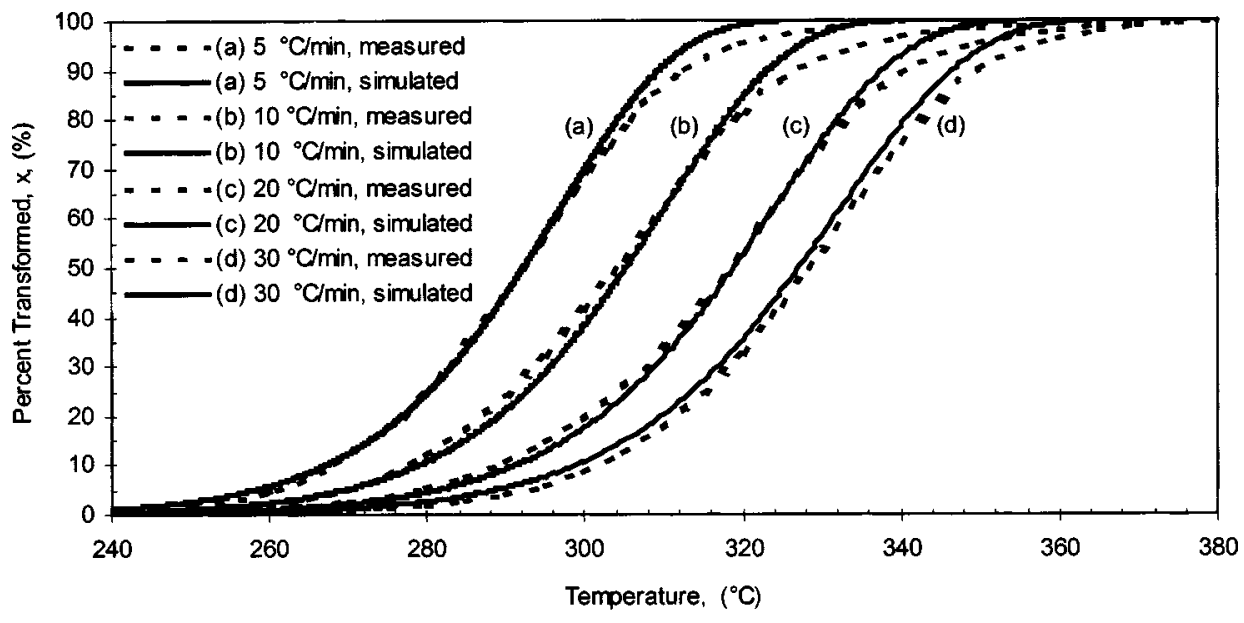

FIG. 9. A comparison of simulated to measured $x$ vs $T$ data. The four sets of curves correspond to constant temperature ramp rates of (a) 5, (b) 10, (c) 20, and (d) $30^{\circ} \mathrm{C} / \mathrm{min}$. couple and send the appropriate power feedback to the furnace. ${ }^{37}$ Therefore, although heat generation may abruptly end in the sample, the heat conduction lag causes a smoothing out of the tail of the transformation peak in the experimental data. Another explanation is post-transformation coarsening. The TEM micrographs showed some evidence of coarsening; this was especially true for the reoriented $L 1_{0}$ particles, which gave way to $L 1_{0}$ particles with a strong (111) texture. The heat given by the release of interfacial energy could also make the peak end less abruptly. Of course, the slight disagreement shown in Fig. 9 could be due to the assumptions of the model as well. The Arrhenian temperature dependence of the rate constant is an approximation. Furthermore, the simulations used a constant Avrami exponent. In reality, the exponent will change with nucleation and growth conditions as the $A 1$ transforms to $L 1_{0} \cdot{ }^{41,45,48} \mathrm{How}-$ ever, the overall fit to the experimental data is good enough to make the application of the JMA analysis to PtMn thin films useful in predicting and modeling transformation behavior.

For practical applications, the ability to simulate isothermal conditions is of considerable interest. Furthermore, it is also necessary to test the ability of the model to predict the phase transformation kinetics under conditions near the limits of its applicability. In other words, the ability of the JMA analysis to model isochronal data from which the $E_{a}, n$, and $K_{0}$ parameters were calculated is not reason enough to believe that it can predict isothermal kinetics at temperatures where the transformation is slow. The results of isothermal simulations of the JMA model are shown in Fig. 10, where they are compared to approximations measured from XRD peak shifts as described earlier. The $260{ }^{\circ} \mathrm{C}$ simulation matches the $260^{\circ} \mathrm{C}$ measured data very well during the beginning stages of the transformation, while the $265^{\circ} \mathrm{C}$ simulation matches the $260^{\circ} \mathrm{C}$ measured data better as the transformation nears completion. Overall, the fit to the $260^{\circ} \mathrm{C}$ data obtained from XRD peak shift analysis (which are approximate) is good enough to consider the JMA model useful in predicting isothermal transformation behavior. This is especially true when considering the reasonable fit to the experimental data despite the reaction's sensitivity to temperature near the onset. In a practical manufacturing process, the anneal designed to induce the phase transformation will be well above the onset, and in this regime the model is expected to better simulate the $A 1-L 1_{0}$ reaction kinetics.

It is worth mentioning that the calculation of the independent parameters used in the JMA analysis were obtained from isochronal experiments. While many isothermal experiments were also performed, their results were not of sufficient quality to be used to calculate these parameters. At temperatures below the onset, the DSC lacked the necessary signal to noise to detect a reliable heat signal from the later, slower stages of the reaction. The characteristic results were lower measured enthalpies of transformation and reactions that appeared to be complete according to the heat signal, but were not complete. A distinct peak was observed in a scanning experiment that was performed after the isothermal signal had gone flat, which verified the presence of further transformation. This problem was also observed by Harmelin et al. in their study of an order-disorder transformation. ${ }^{49} \mathrm{At}$ temperatures near and above the onset, a typical problem $^{26,44,49}$ with isothermal experiments was observed. The reaction possesses a short delay time and therefore the

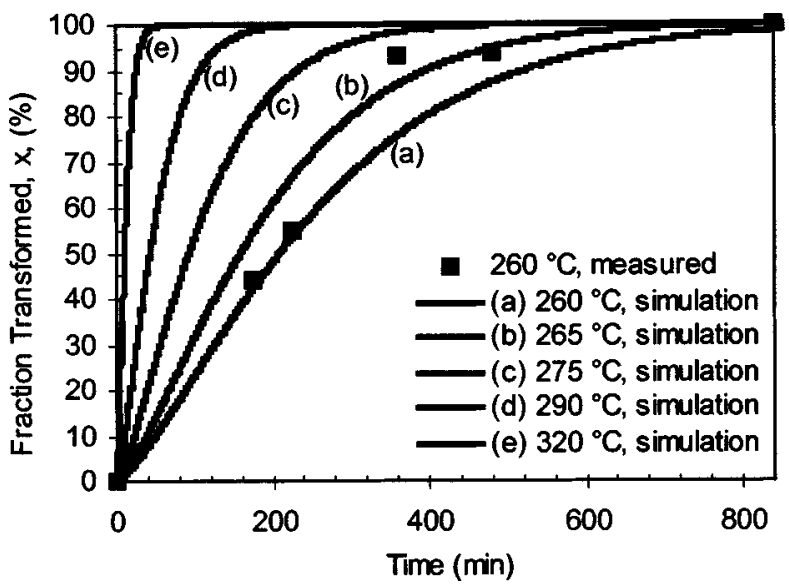

FIG. 10. A comparison of simulated to measured $x$ vs $t$ data. The $260^{\circ} \mathrm{C}$ simulation, curve (a), matches the measured $260{ }^{\circ} \mathrm{C}$ approximations from measured data very well during the beginning stages of the transformation, while the $265^{\circ} \mathrm{C}$ simulation, curve (b), matches the $260^{\circ} \mathrm{C}$ data better as the transformation nears completion. 
DSC is not able to stabilize at the experimental temperature before the reaction proceeds. The results from these experiments show significant artifacts near the beginning of the transformation, leading to imprecise measurements of the transformation enthalpy and Avrami exponent. Finding a happy medium between these regions was not possible and ultimately no isothermal DSC data was directly used to calculate the parameters for the JMA analysis.

Nonetheless, some information was extracted from isothermal DSC experiments, mainly to crosscheck the data used in the analysis. The Avrami exponent was calculated from scans at $250^{\circ} \mathrm{C}$ and below by taking the slope from $\ln [-\ln (1-x)]$ vs $\ln (t)$ plots. ${ }^{20,41,45}$ Although the linear correlation coefficients from the plots were all 0.99 or higher, there was a significant variance in the data as the calculated $n$ values ranged from 1.08 at $250{ }^{\circ} \mathrm{C}$ to 1.69 at $230^{\circ} \mathrm{C}$. However, the average calculated Avrami exponent was 1.34, very similar to the value obtained from the Ozawa analysis of isochronal experiments. Although obtaining quantitative data from isothermal experiments were unreliable, there is an important qualitative assessment that was verified in all of the isothermal scans. The presence of a definite peak, in contrast to a monotonically decreasing signal, was present in all isothermal scans. As discovered by Chen and Spaepen, the presence of an isothermal peak is evidence (although not definitive proof) that the analyzed DSC peaks represent a nucleation and growth transformation and is proof that they do not represent normal grain growth from pre-existing nuclei. $^{28,29,40,50}$

\section{CONCLUSIONS}

Sputter deposited PtMn thin films used in spin valves are found to possess a metastable, chemically disordered, paramagnetic $A 1$ structure. Investigation of the phase diagram suggests an $L 1_{0}$ structure should be the equilibrium phase at temperatures under $900^{\circ} \mathrm{C}$. Upon subsequent annealing, the PtMn films undergo a phase transformation to obtain that stable, chemically ordered, antiferromagnetic, $L 1_{0}$ structure. Experiments have revealed that the kinetics of this transformation became favorable around $260^{\circ} \mathrm{C}$ and the enthalpy of the transformation is exothermic, with a magnitude of $-12.1 \mathrm{~kJ} / \mathrm{mol}$ of atoms.

The TEM image analysis suggests that $L 1_{0}$ clusters may preferentially nucleate at nanotwin defects and grain boundaries in the $A 1$ phase. A small amount of reorientation from the as-deposited (111) texture is observed in intermediate stages, but it is later consumed by (111) textured $L 1_{0}$ phase. The TEM images also suggest that the majority of the $L 1_{0}$ PtMn phase forms by nucleating plates which predominantly propagate parallel to the film surface until impeded by defects or other transformed regions. The $L 1_{0}$ phase then predominantly grows laterally from these plates. The process can also occur along the other two (111) symmetry directions, but in this case the twin planes grow to the interfaces with $\mathrm{Ni}-\mathrm{Fe}$ and the seed layer, and the majority of twinning occurs laterally across the film. With further annealing, the $L 1_{0}$ particles grow in size through coarsening.
An analytical description of the phase transformation was obtained with the addition of the JMA analysis. The Kissinger and Ozawa methods were used to determine the kinetic parameters from isochronal DSC experiments. The calculated values for the activation enthalpy, Avrami exponent and rate constant were $E_{a}=131.5 \mathrm{~kJ} / \mathrm{mol}, n=1.31$, and $\ln K_{0}=19.95$, respectively. The model was then used to simulate isochronal fraction transformed versus temperature with good agreement to experimental data. The ability of the model to predict isothermal kinetics was then tested by a simulation comparison to data calculated from XRD peak shifts. The simulations displayed the correct trend and reasonable agreement to the calculated data. It is therefore concluded that the transformation behavior of these PtMn films can be adequately predicted by JMA kinetics, provided that the temperature of interest is near or above the onset temperature of $260^{\circ} \mathrm{C}$.

\section{ACKNOWLEDGMENTS}

P.F.L. would especially like to thank Professor John Perepezko for insight on phase transformation theory and Chris Pelto for his review of this manuscript. Financial support for this research was provided by Seagate Technology and the Division of Materials Sciences, Office of Basic Energy Research of DOE through Grant No. DE-FG0299ER45777.

${ }^{1}$ M. Baibich, J. Broto, A. Fert, F. Nguyen Van Dau, and F. Petroff, Phys. Rev. Lett. 61, 2472 (1988).

${ }^{2}$ P. Levy, Solid State Phys. 8, 368 (1995).

${ }^{3}$ B. Dieny, V. Sperioso, S. Parkin, B. Gurney, D. Wilcoit, and D. Mauri, Phys. Rev. B 43, 1297 (1991).

${ }^{4}$ C. Tsang, R. Fontana, T. Lin, D. Heim, V. Speriosu, B. Gurney, and M. Williams, IEEE Trans. Magn. 30, 6 (1994).

${ }^{5}$ M. J. Carey and A. E. Berkowitz, Appl. Phys. Lett. 60, 3060 (1992).

${ }^{6}$ S. Yuan and H. Bertram, J. Appl. Phys. 75, 6385 (1994).

${ }^{7}$ J. C. Mallinson, Magnetio-Resistive Heads, Fundamentals and Applications (Academic, San Diego, CA, 1996).

${ }^{8}$ M. Lederman, IEEE Trans. Magn. 35, 794 (1999).

${ }^{9}$ P. F. Ladwig, I. Tsu, C. H. Chang, and Y. A. Chang, IEEE Trans. Magn. 37, 1132 (2001)

${ }^{10}$ T. Lin, D. Mauri, N. Staud, C. Hwang, J. K. Howard, and G. L. Gorman, Appl. Phys. Lett. 65, 1183 (1994).

${ }^{11}$ A. J. Devasahayam and M. Kryder, IEEE Trans. Magn. 32, 4654 (1996).

${ }^{12}$ S. Mao, S. Gangopadhyay, N. Amin, and E. Murdock, Appl. Phys. Lett. 69, 3593 (1996).

${ }^{13}$ H. Kishi, Y. Kiade, Y. Miyake, A. Tanaka, and K. Kobayashi, IEEE Trans. Magn. 32, 3380 (1996).

${ }^{14}$ G. W. Anderson, Y. Huai, L. Miloslavsky, C. Qian, and Y. Feng, J. Appl. Phys. 85, 6109 (1999).

${ }^{15}$ R. F. C. Farrow, R. F. Marks, S. Gider, A. C. Marley, S. S. P. Parkin, and D. Mauri, J. Appl. Phys. 81, 4986 (1997).

${ }^{16}$ K. Krishnan, C. Nelson, C. Echer, R. F. C. Farrow, R. F. Marks, and A. J. Kellock, J. Appl. Phys. 83, 6810 (1998).

${ }^{17}$ M. Saito, Y. Kakihara, T. Watanabe, and N. Hasegawa, J. Magn. Soc. Jpn. 21, 505 (1996).

${ }^{18}$ M. Saito, N. Hasegawa, F. Koike, H. Seki, and T. Kuriyama, J. Appl. Phys. 85, 4928 (1999).

${ }^{19}$ H. Nagai, M. Ueno, and F. Hikami, IEEE Trans. Magn. 35, 5 (1999).

${ }^{20}$ C. Michaelson, K. Barmak, and T. P. Weihs, J. Phys. D 30, 3167 (1997).

${ }^{21}$ W. Mahler, Z. Metallkd. 46, 282 (1955).

${ }^{22}$ J. H. Perepezko, Mater. Res. Soc. Symp. Proc. 19, 223 (1983).

${ }^{23}$ D. L. Smith, Thin Film Deposition, Principles and Practice (McGrawHill, New York, 1995).

${ }^{24}$ F. R. de Boer, R. Boom, W. C. M. Mattens, A. R. Miedema, and A. K. Niessen, Cohesion in Metals, Transition Metal Alloys (Elsevier, Amsterdam, 1988). 
${ }^{25}$ R. Krachler, H. Ipser, and K. L. Komarek, Z. Metallkd. 79, 96 (1988).

${ }^{26}$ D. W. Henderson, J. Non-Cryst. Solids 30, 301 (1979).

${ }^{27}$ H. Yinnon and D. R. Uhlmann, J. Non-Cryst. Solids 54, 253 (1983).

${ }^{28}$ L. C. Chen and F. Speaepen, J. Appl. Phys. 69, 679 (1991).

${ }^{29}$ F. Spaepen and C. V. Thompson, Appl. Surf. Sci. 38, 1 (1989).

${ }^{30}$ W. A. Johnson and K. F. Mehl, Trans. Am. Inst. Min., Metall. Pet. Eng. 135, 315 (1981).

${ }^{31}$ M. Avrami, J. Chem. Phys. 7, 1103 (1939).

${ }^{32}$ M. Avrami, J. Chem. Phys. 8, 212 (1940).

${ }^{33}$ M. Avrami, J. Chem. Phys. 9, 177 (1941)

${ }^{34} \mathrm{~J}$. W. Christian, The Theory of Transformations in Metals and Alloys, 2nd ed. (Pergamon, New York, 1975).

${ }^{35}$ R. A. Ristau, K. Barmak, L. H. Lewis, K. R. Coffey, and J. K. Howard, J. Appl. Phys. 86, 4527 (1999).

${ }^{36}$ Q. Z. Hong, K. Barmak, S. Q. Hong, and L. A. Clevenger, J. Appl. Phys. 74, 4958 (1993).

${ }^{37}$ P. D. Garn, Crit. Rev. Anal. Chem. 2, 65 (1972).
${ }^{38}$ H. E. Kissinger, J. Res. Natl. Bur. Stand. 57, 217 (1956).

${ }^{39}$ P. Altuzar and R. Valenzuela, Mater. Lett. 11, 101 (1991).

${ }^{40}$ L. C. Chen, F. Spaepen, J. L. Robertson, S. C. Moss, and K. Hiraga, J. Mater. Res. 5, 1871 (1990).

${ }^{41}$ S. Budurov and G. Zolova, Cryst. Res. Technol. 28, 925 (1993).

${ }^{42}$ S. Ranganathan and M. von Heimendal, J. Mater. Sci. 16, 2401 (1981).

${ }^{43}$ J. H. Perepezko (private communication).

${ }^{44}$ T. Ozawa, Polymer 12, 150 (1971).

${ }^{45}$ C. W. Price, Acta Metall. Mater. 38, 727 (1990).

${ }^{46}$ K. Barmak, J. M. Rickman, and C. Michaelsen, J. Electron. Mater. 26, 1009 (1997).

${ }^{47}$ C. Michaelsen, S. Wohlert, R. Bormann, and K. Barmak, Mater. Res. Soc. Symp. Proc. 398, 245 (1996).

${ }^{48}$ K. Sekimoto, Physica A 135, 328 (1986).

${ }^{49}$ M. Harmelin, S. Lefebvre, M. Bessiere, and Y. Calvayrac, Thermochim. Acta. 125, 59 (1988).

${ }^{50}$ L. C. Chen and F. Spaepen, Nature (London) 336, 366 (1988). 